\title{
علاقة إقليم جنوب غرب شبه الجزيرة العربية بشرق أفريقيا حتى نهاية العصر الحجري الحديث
} محمد طه محمد الأمير باحث دكتوراه ـ قسم التاريخ والحضارة ـ كلية اللغة العربية بالقاهرة - جامعة الأزهر - مصر alamer2951982@gmail.com

ا.م.د/ أحمد رفعت عبد الجواد

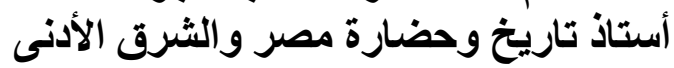

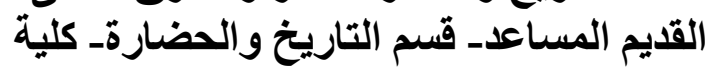

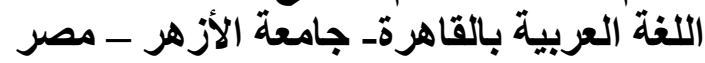
dr.ahmed.refat2020@gmail.com
ا.د/ عائشة محمود عبد العال

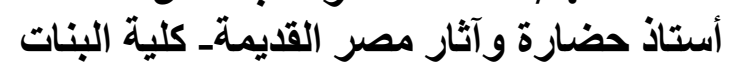

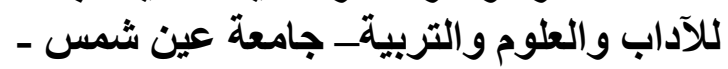
مصر

Aisha_abdelaal@women.asu.edu.eg

أ.د/ طلعت أحمد محمد عبده

أستاذ الجغرافيا الطبيعيةـ قسم التاريخ والحضارة- كلية اللغة العربية بالقاهرة

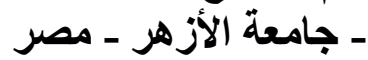

\section{Talaat abdo@gmail.com}

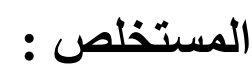

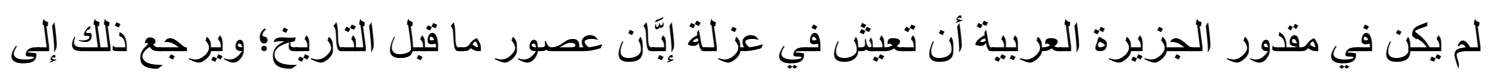

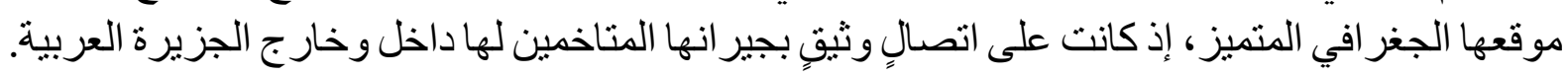

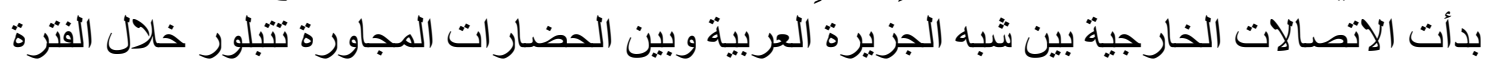

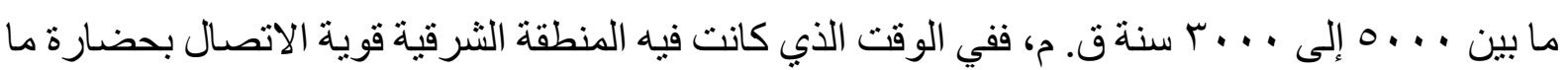

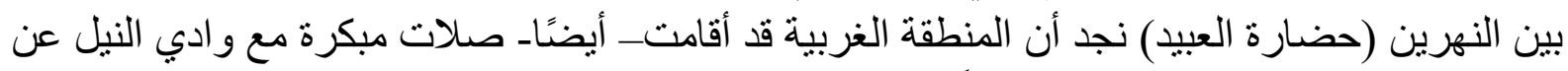

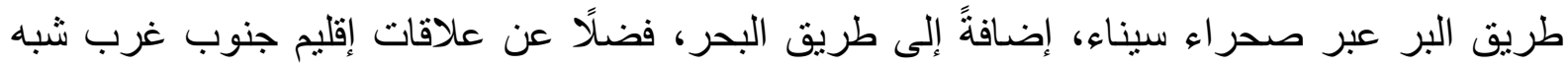
الجزيرة العربية مع شرق أفريقيا.

وقد أخذت المعطيات الأثرية تتو افق في فترة العصر الحجري القديم بمرحلتيه الأولدو انية والآشولية بين إقليم جنوب غرب شبه الجزيرة العربية مع ما هو معروف في شرق أفريقيا، حيث وجدت فئ فيها شو اهد الهد

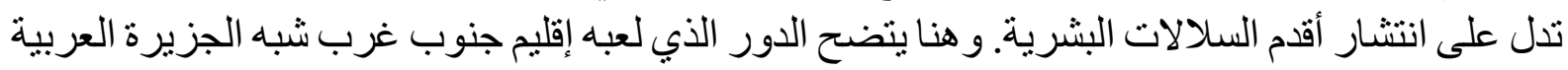

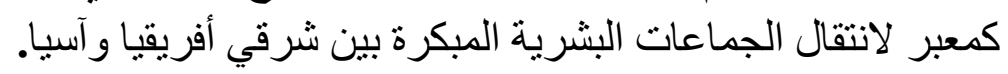

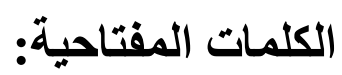
شرق أفريقيا ــ العصر الحجريـ الجزيرة العربية ــ الحضـارة الأفريقية. 


\section{أولًا: أهمية موقع شبه الجزيرة العربية وشرق أفريقيا:}

يشغل إقليم جنوب غرب شبه الجزيرة العربية من منظور الجغر افيا الحيوية و العمليات التطورية مكانة

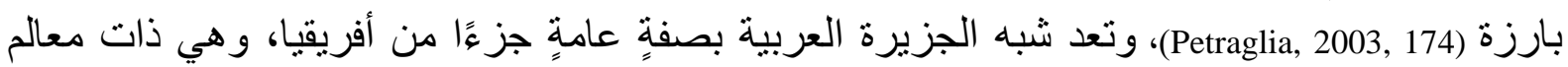
جيولوجية ومناخية مميزة (Tosi, 1986, 463) ، كما برنبط الهامش الثرقي لساحل البحر الأحمر بتاريخ جيولوجي طويل، وذلك بدءًا من ترسب ما قبل الكمبري، حيث الصخور الرسوبية و البركانية, Brown).

1970, 76)

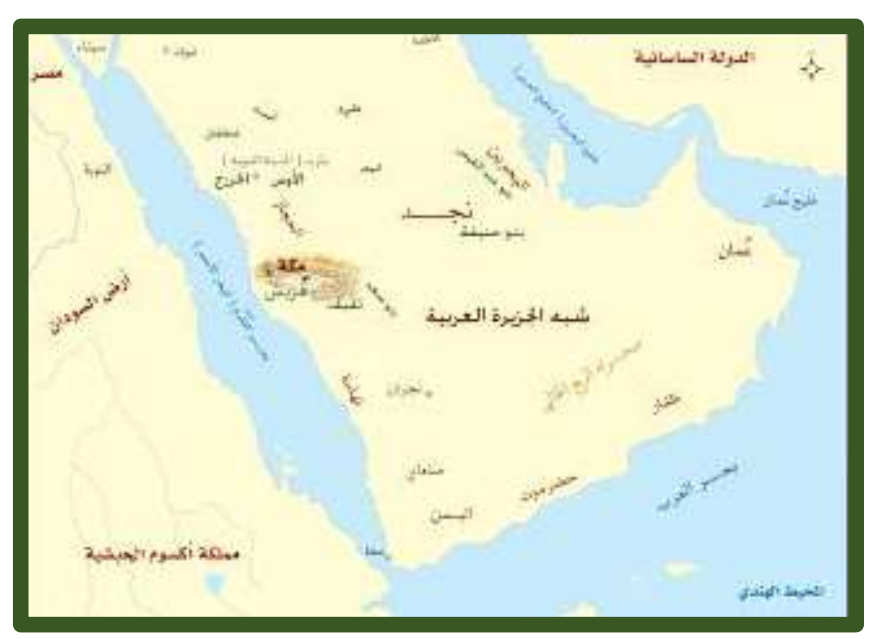

توضح موقع إقليم شرق أفريقيا المقابل لجنوب غرب شب شبه الجزيرة العربية على الخريطة

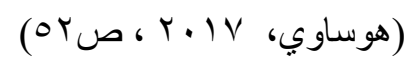

ومن ناحيةٍ أخرى فتقع شبه الجزيرة العربية في أقصى جنوب غربي آسيا، وقد كان لهذا الموقع أهميةً

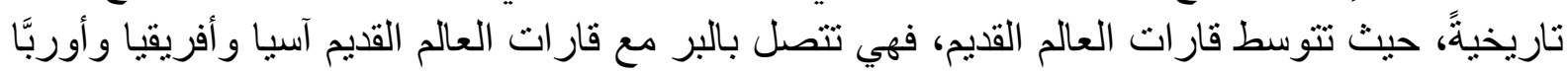

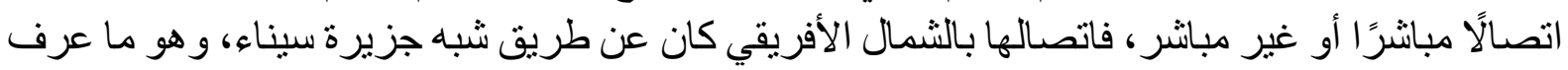

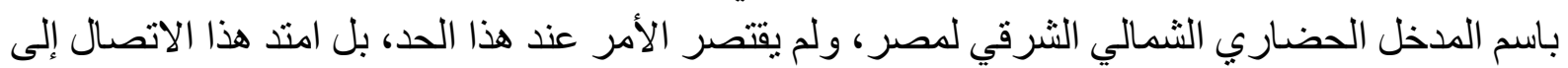

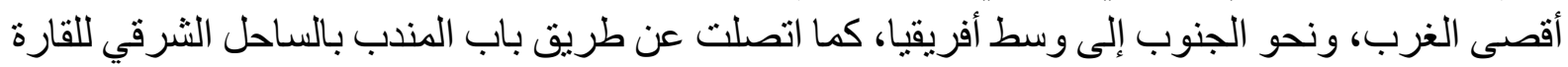

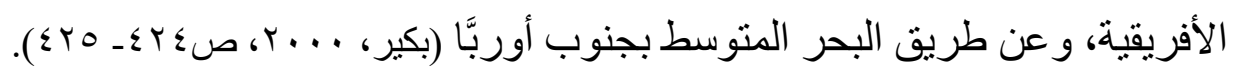

ولهذا كان لها ميزة الاتصال الحضاري أو المكاني في أطر افها الثمالية والجنوبية، بحيث كانت

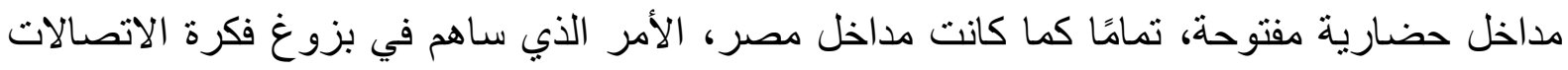

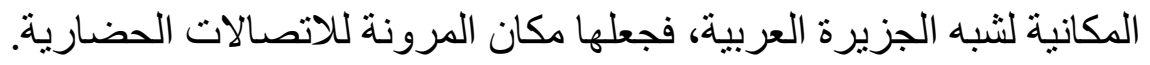

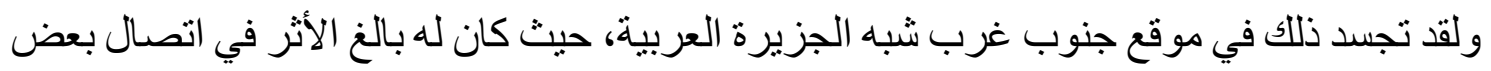

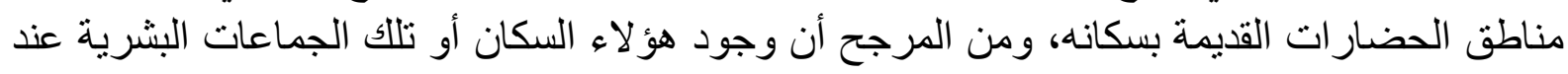

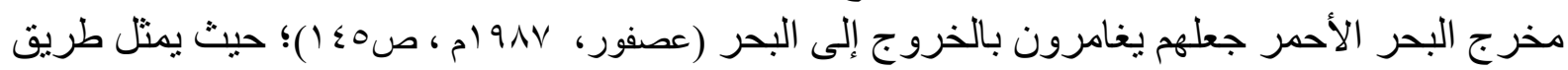

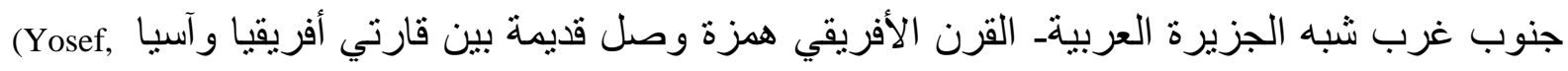

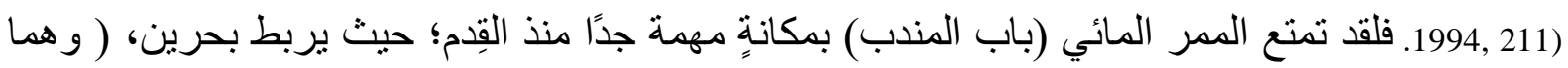
(بحر العرب) و(البحر الأحمر)، ويابسين وهما (شبه الجزيرة العربية عند رأس المنهال) و(شرق أفريقيا

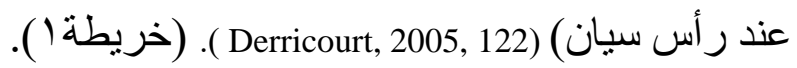


لقد اقتصرت عملية الاتصال بين القار ات قديمًا- من خلال المفهوم الجغر افي البيئي- على الممرات

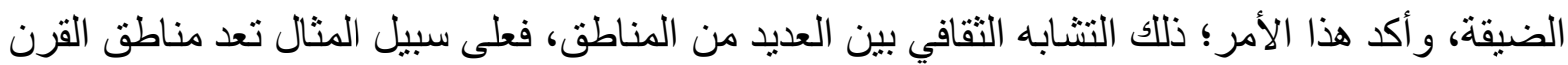

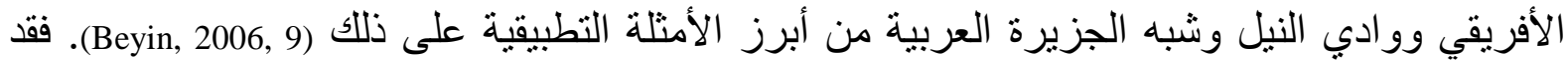

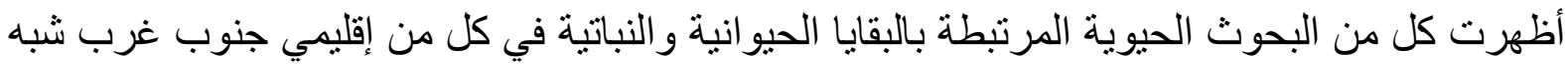
الجزيرة العربية وشرق أفريقياوجود العلاقات التي تمت بين الإقليمين عن طريق الممر الرئيس من إثيوبيا

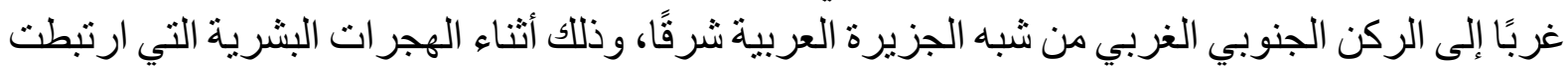

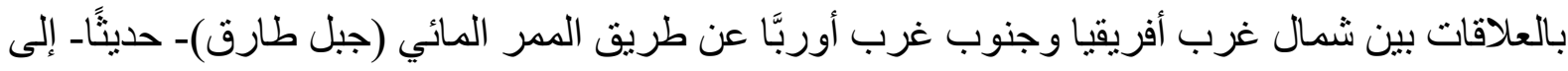

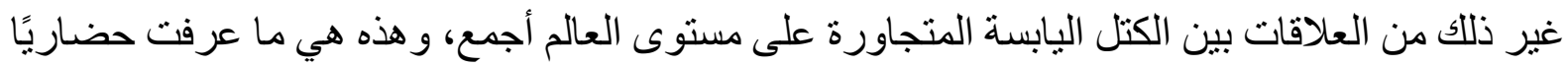
باسم المعابر الأرضية Land Bridges فيما بين قار ات العالم القديم و الجديد (Yosef, 1987, 31) .

أما منطقة شرق أفريقيا فتعد منطقة تجمع عمر اني بشري، وهي تشبه في ذللك الأمر إقليم بلاد الشام.

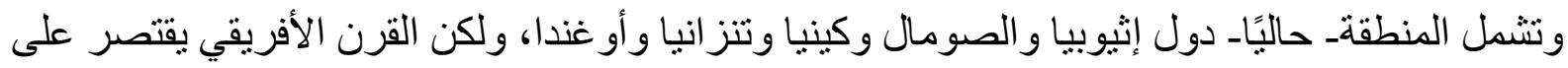

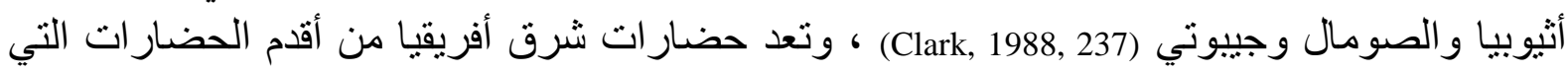

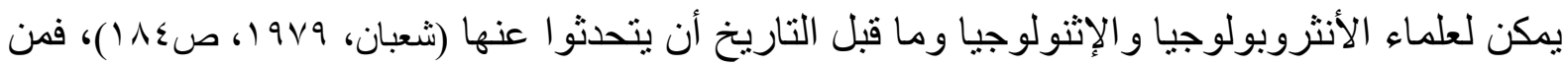

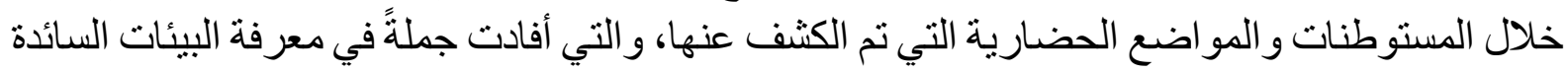

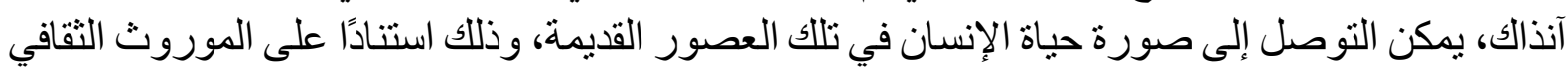
للإنسان في تلك العصور (Beinart, 2003, 85).

ويعد التاريخ الحضاري للقارة الأفريقية عامةً وساحلها الشرقي خاصةً من الأهية بمكان، فقد

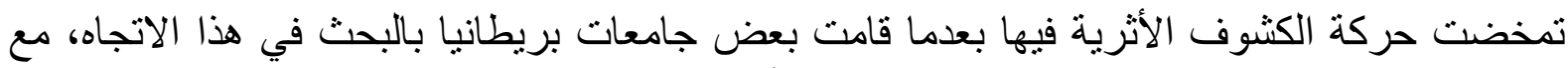

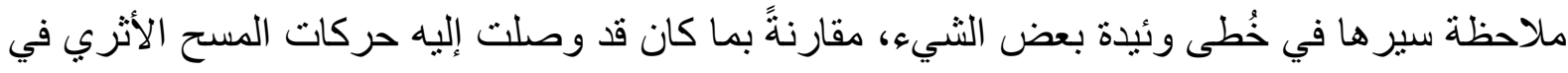

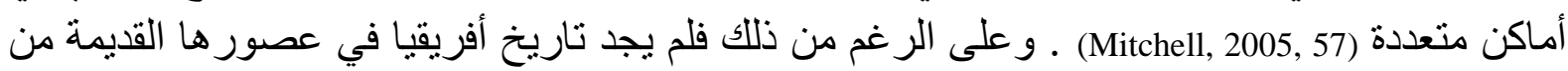
الاهتمام الذي يليق به كتاريخ قارة من أقدم قار ات العالم القديم، فقد غطى تاريخ الاستعمار الأوروبي لها

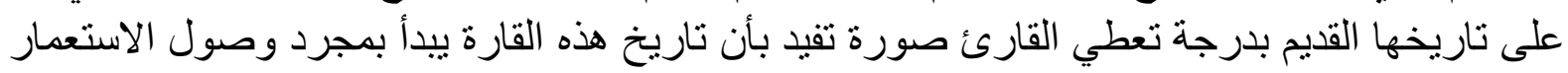

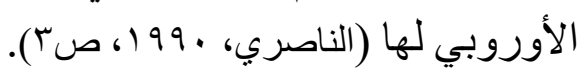

ومما جعل سو احل أفريقيا الشرقية مقصد العرب قديمًا مجاورتها لها، فضلًا عن ارتباطها بحركات

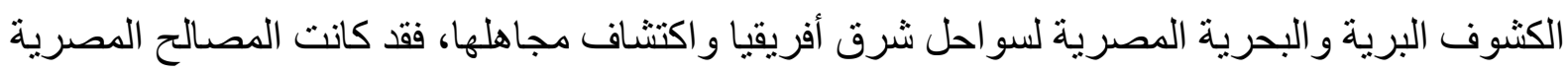

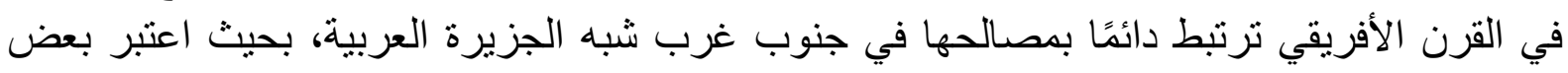

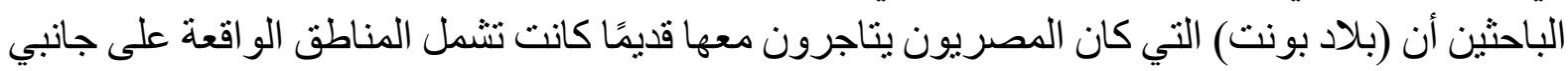

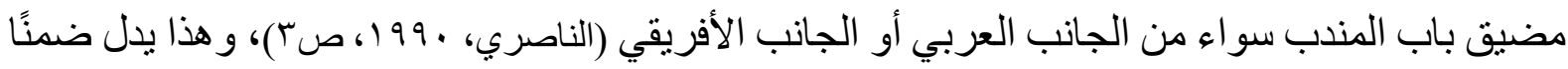
على مدى اتصال جنوب غرب شبه الجزيرة العربية بشرق أفريقيا ووحدتهما الإقليمية. ثانيًا: الاتصال بين شبه الجزيرة العربية وشرئ أنرق أفريقيا:

تتمتع شبه الجزيرة العربية بصلة حضارية وثيقة بالجانب الثرقي لقارة أفريقيا الذي يقابلها، فالثبه

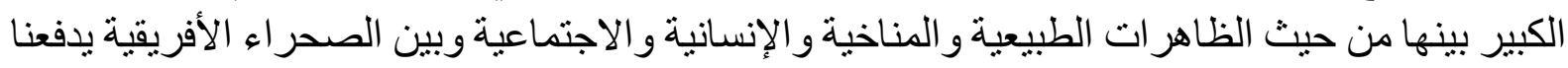




\section{علاقة إقليم جنوب غرب شبه الجزيرة العربية بشرق أفريقيا حتى نهاية العصر الحجري الحديث}

إلى تسمية هذه المنطقة بـــ (أفريقيا الآسيوية) وحفرة البحر الأحمر الكبيرة التي تفصل القارة الأفريقية عن

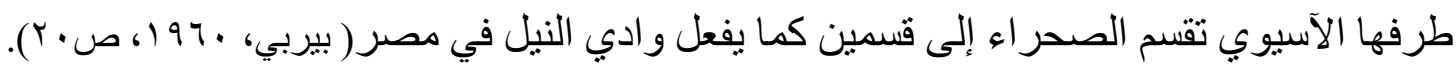
و هناك توجه يذكر بأن أقدم اكتشاف لإنسان العصر الحجري القديم في شرق أفريقيا وتحديدًا في

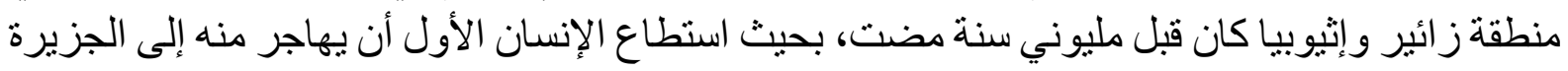

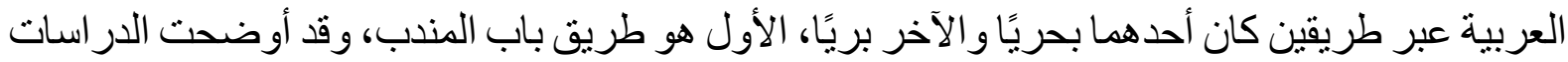

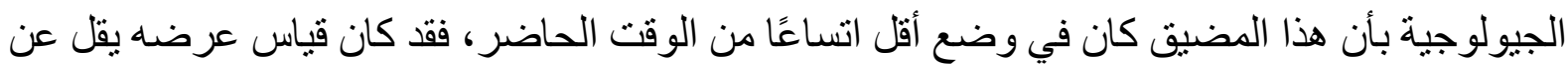

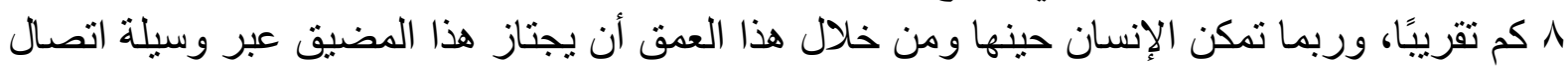

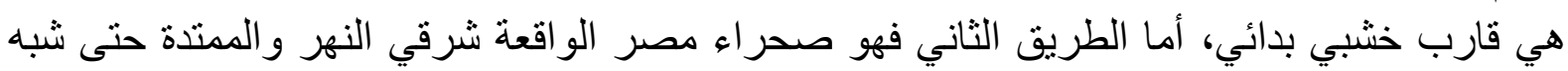

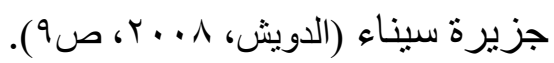

وقد تمكنت الجماعات البشرية خلال تلك المرحلة من صناعة أدوات الحرب و القتال من موادٍ

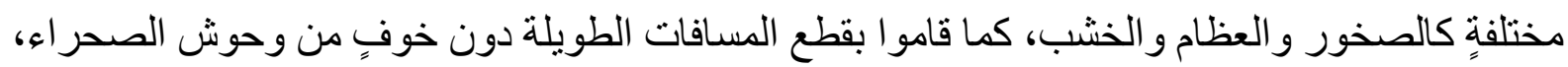
و هذا ما يؤكد افتر اضات ظهور مواقع كثثرة على هذا الطريق تعود في تأريخها إلى الحقبة الآشولية، كما

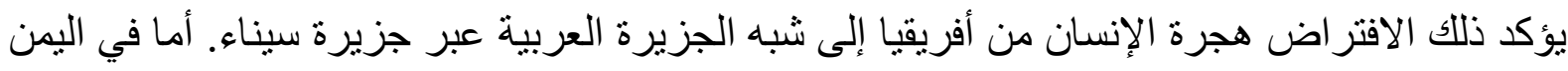

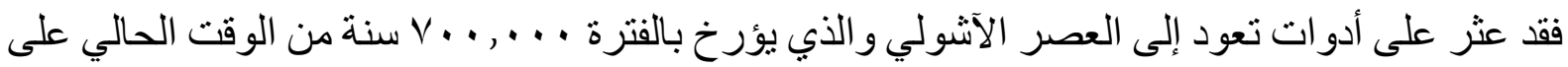

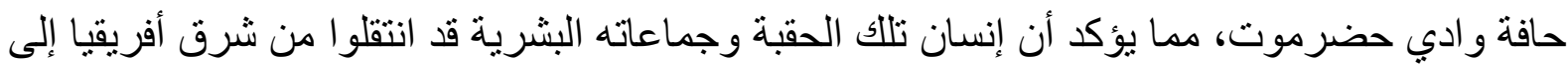

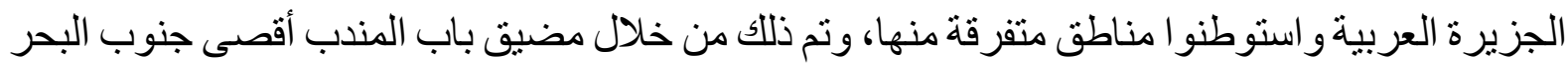

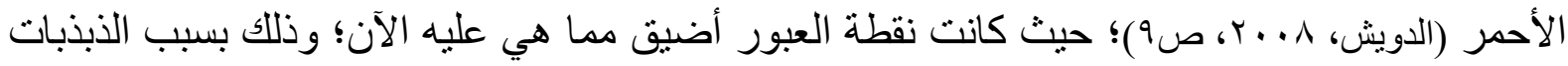

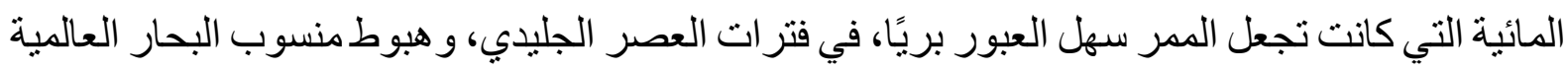

آنذالك (Andrew, 2011, 387).

إن الآثار التي كثف عنها في مناطق متعددة في جنوب وجنوب غرب شبه الجزيرة العربية و التي

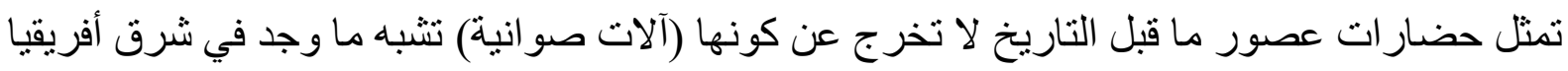
من العصر الحجري القديم، الأمر الذي أدى إلى افتر اض اضث نظريتين:

النظرية الأولى: نشير إلى أن الحضارة انتقلت من شبه الجزيرة العربية إلى الساحل الأفريقي.

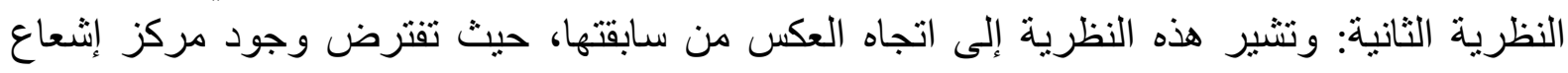

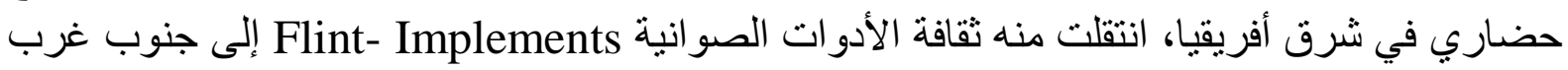

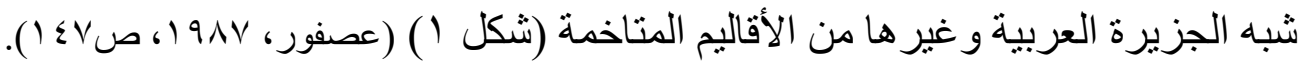

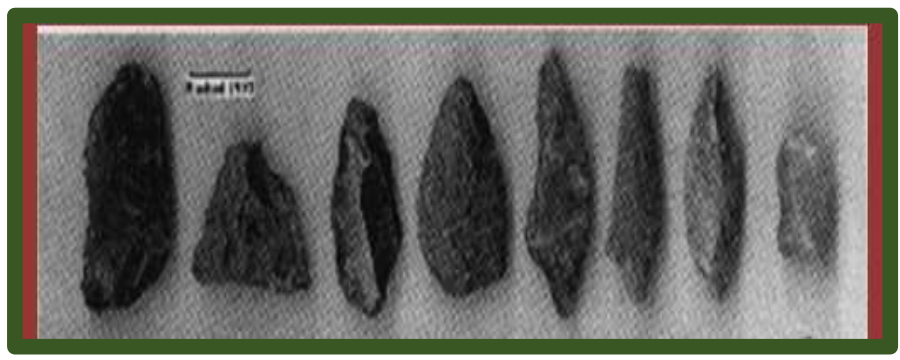

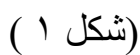

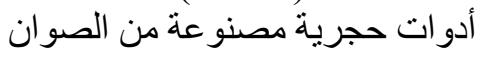

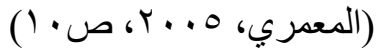




\section{علاقة إقليم جنوب غرب شبه الجزيرة العربية بشرق أفريقيا حتى نهاية العصر الحجري الحديث}

و هنا تجدر الإشارة إلى أن حضار ات الساحل الشرقي لأفريقيا قد مرت في عصور ها الحجرية بالعديد

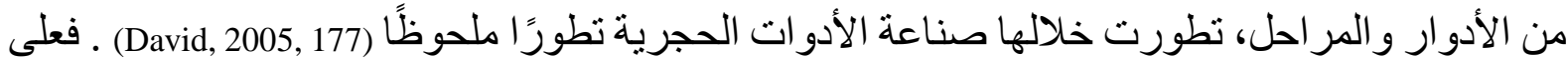

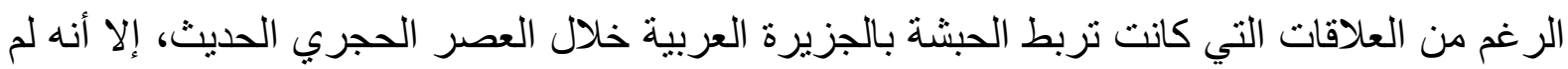
يعثر على مخلفات تؤكد صحة هذا الاعتقاد بالقدر الكافِ، سوى ما وُجد في منطقة القرن الأفريقي؛ وربما يرجع السبب في ذلك إلى أن الوقت الذي حدثت فيه الهجر ات النيوليثية من آسيا، كان فيه الجفاف يسيطر

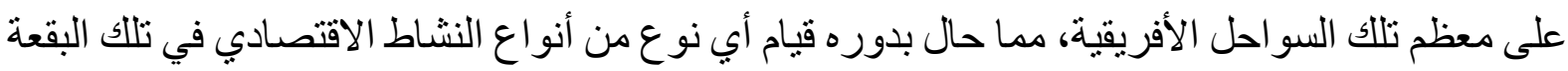

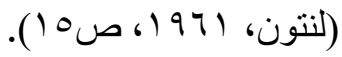

وبعد انتهاء فترة البلايستوسين بدأت الجماعات البشرية في شرق أفريقيا ثُرسي دعائم الحياة البشرية

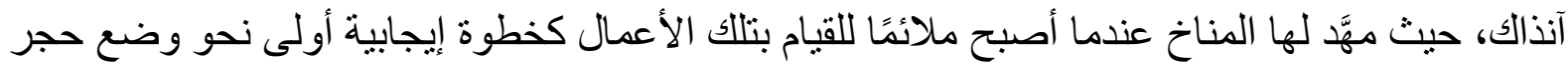

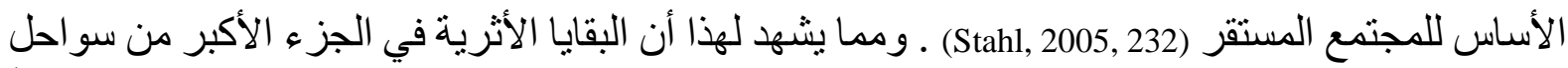
شرق أفريقيا دلت على أن هذه المجتمعات قد عرفت الزر اعة، وصناعة الفخار في وقتٍ مبكرٍ، خاصةً سو احل تنز انيا، وهو ما عرفته الدر اسات الأركيولوجية باسم صناعة المستأنسات The domesticated

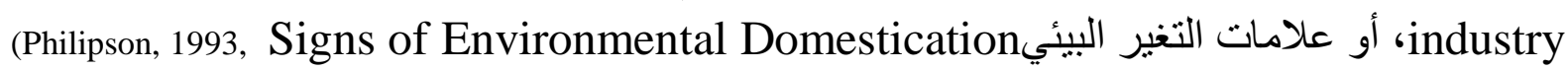

ومن الأمور المشكوك فيها أن جميع سكان شرق أفريقيا إبَّان العصر الحجري الحديث قد اعتمدوا

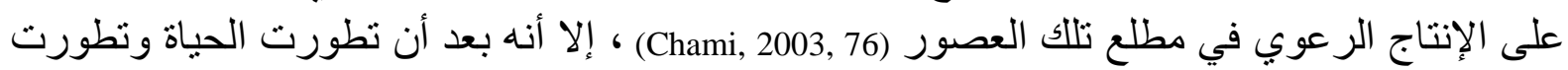
معها الطرق الفنية اللازمة لقيام الاقتصاد الرعوي الري عندهم، بدأت الهجر ات من الجزيرة العربية تشق طريقها

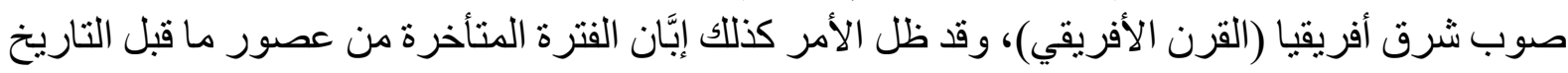

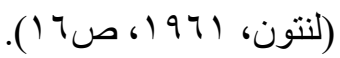

\section{ثالثًا: جوانب الاتصال الحضاري:}

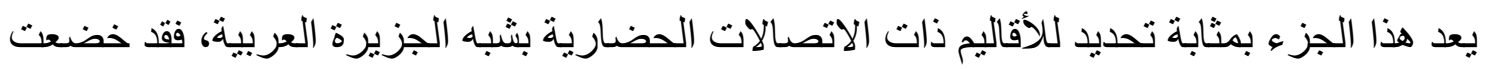

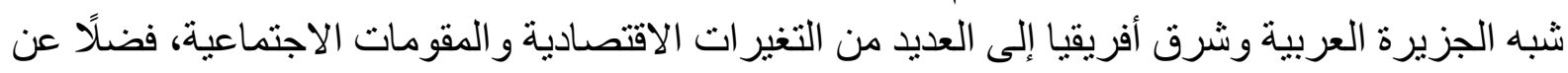

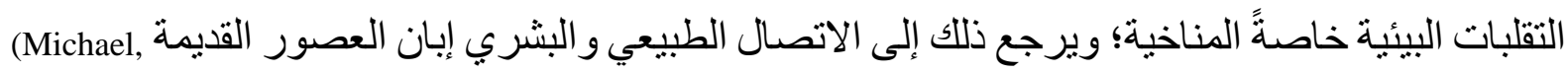

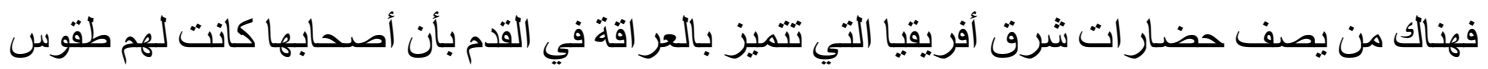

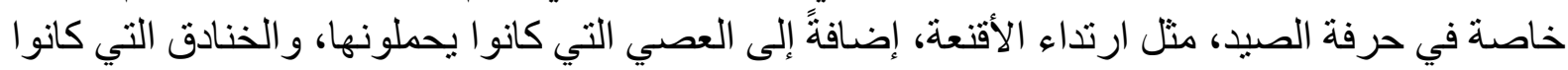

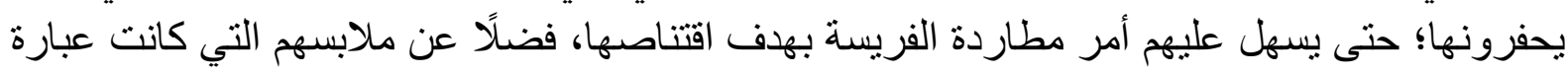
عن مآزر من الجلا و أغطية تستر العورة (Baumann, 1940, 131 ).

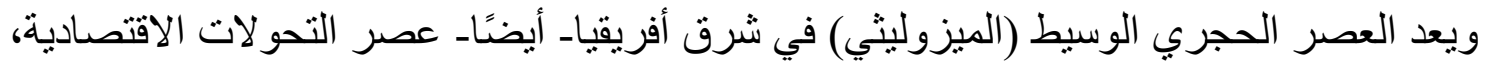
وذلك مثل بقية أنحاء العالم القديم، حيث تحول الإقليم من الاقتصاد القائم على الحرف الأفئي الأولية أو البدائية

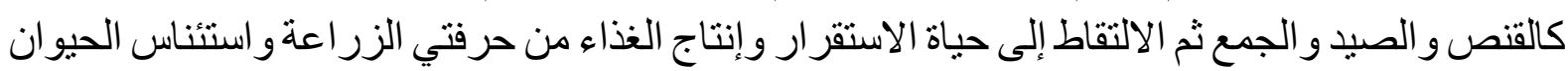

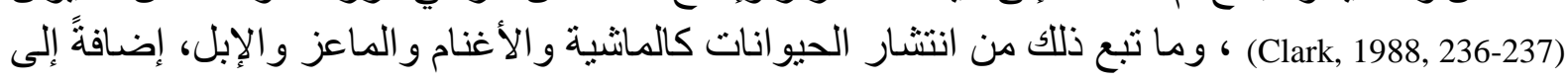




\section{علاقة إقليم جنوب غرب شبه الجزيرة العربية بشرق أفريقيا حتى نهاية العصر الحجري الحديث}

(Chami, بعض أنو اع الطيور وذللك مثل الحيو انات ذاتها المنتشرة في إقليم جنوب غرب شبه الجزيرة العربية

وقد تطورت عمليات الرعي إبَّن العصر الحجري الحديث في شرق أفريقيا إلى درجة متقدمة، نتج

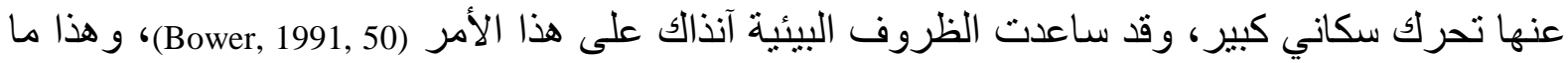
يدعو إلى القول: بأنه ارتبط التطور الاجتماعي والثقافي في إقليم شرق أفريقيا إلا بعد سيادة العصر الحجري الحئ

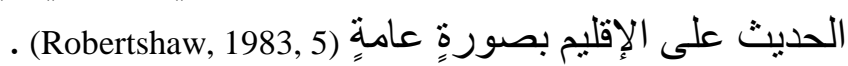

ويرى بعض علماء الحفريات أن الحضار ات الأفريقية في العصر الحجري الحديث قد نبعت أصولها

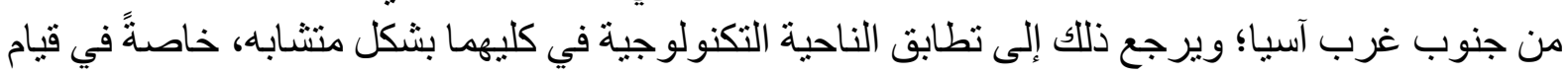

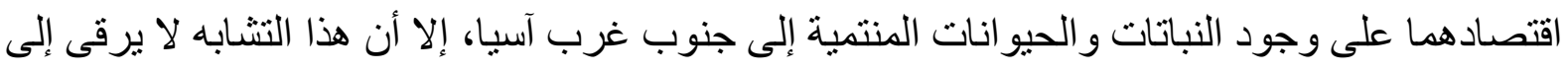

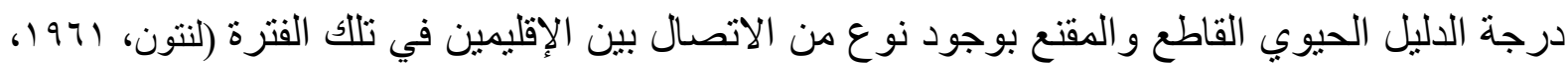

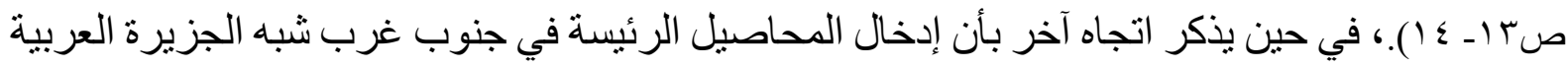

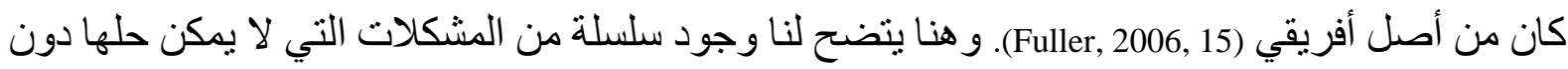

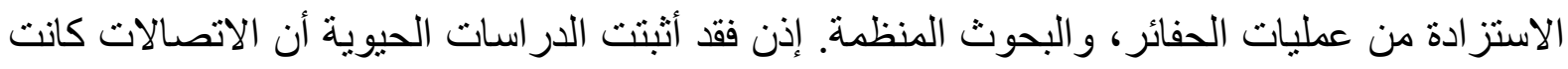

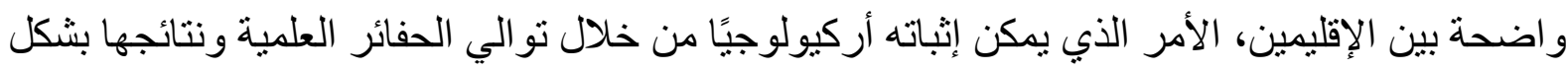

منتظم.

و ما يهمنا في هذا السبيل ليس من كانت له قدم السبق في نشأة الحضارة عنده أولًا، ومن ثنمّ تصدير ها إلى الجانب الآخر، بل الثاهد في هذه القضية برمتها هو هل كانت هنالك علاقة حضارية بين إقليم جنوب

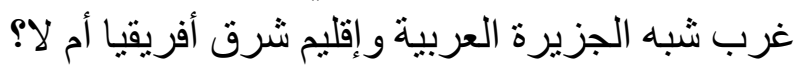

وللإجابة على هذا التساؤل يمكن القول: بأنه من خلال ما تقدم يتضح لنا التشابه القوي بين سكان إقليم جنوب غرب شبه الجزيرة العربية وسكان مناطق شرق أفريقيا، من زو ايا فنون مرحلة حياة الجمع و الالتقاط

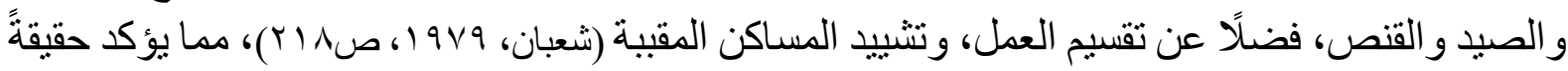

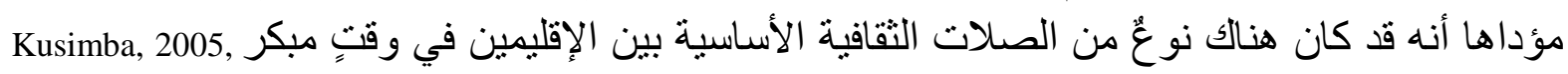

ولعل السبب الرئيس في ذلك يكمن في ظروف أخرى بعيدة عن الاتصال التقافي أو الهجرات

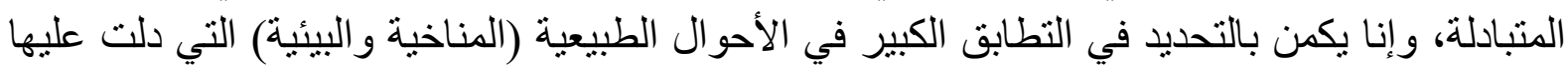

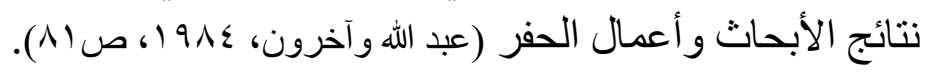

وجاء هذا الاستتناج نتيجة تحليل علمي للتربة المصاحبة للطبقات الترسبية في مو اقع الإقليمين، حيث

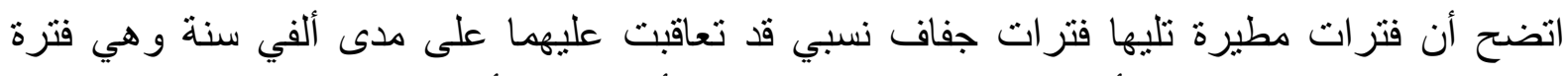

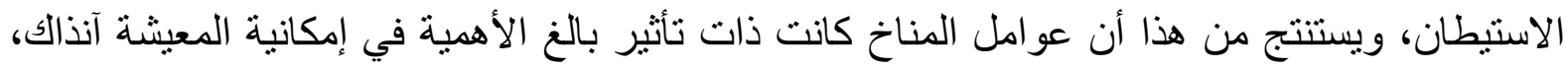

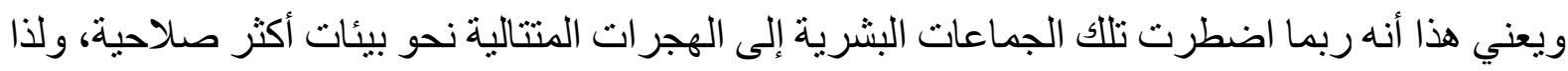
فمن المحتمل أن أقوام العصور الحجرية في تللك الأقاليم قد عاثوا تحت التهرية وطأة أحوال مناخية وبيئية اضطرتهم للارتحال الموسمي أو شبه الموسمي إلى مناطق شمالية أكثر ملاعمة للعيش(عبد الله وآخرون،

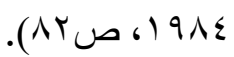




\section{علاقة إقليم جنوب غرب شبه الجزيرة العربية بشرق أفريقيا حتى نهاية العصر الحجري الحديث}

وخلاصة القول: فإن در اسات (فيشر) التي نشرت في كتابه الموسوم بالثرق الأوسط، والذي صدر عام 9 ا ام، تؤكد ببساطة شديدة وجود ارتباط واضح بين جنوب غرب شبه الجزيرة العربية وشرق أفريقيا، وذلك من خلامل ما يلي:

• أنه ذكر أن الإقليمين كأنهما إقليم طبيعي و احد، تم انفصاله إلى قسمين.

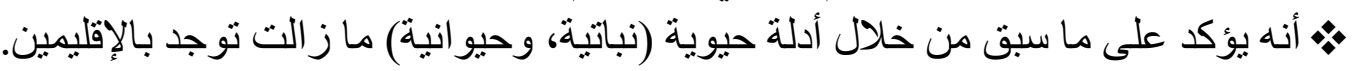

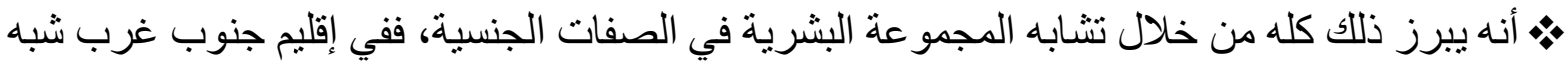
الجزيرة العربية توجد مجمو عات بشرية تحمل الملامح الخاصة بالمجمو عة الزنجية كلون البشرة، وشكل

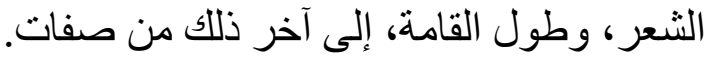
• إن المجموعات البشرية ذات مساكن من نوع خاص (مساكن مشيدة من الأفرع النباتية أو غصون

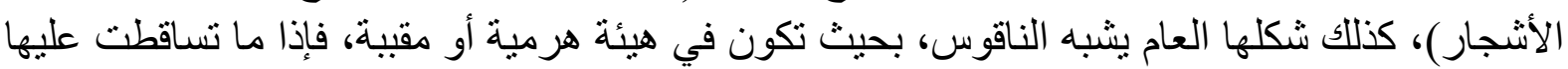
الأمطار انسابت على أطر افها وتجمعت في أوعية فخارية لأغر اض الثرب الثرب أو الأغر اض المنزلية كطهي الطعام. •) وجود الحيو انات المتسلقة (كالقردة من نوع البابون)، و هي تنيع في جنوب غرب شبه الجزيرة العربية،

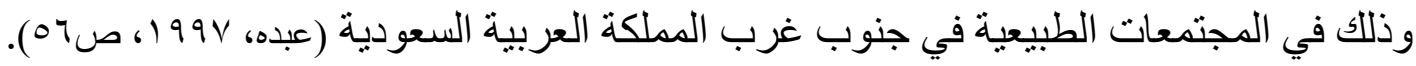

\section{رابعًا: نماذج الاتصال الحضاري:}

ربما كانت هنالك ثقافتان فقط ممثلتان في شرق أفريقيا، وهما: ثقافة العصر الحجري القديم الأدنى،

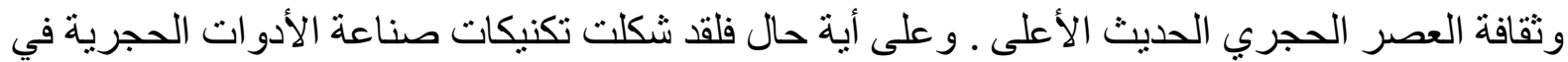

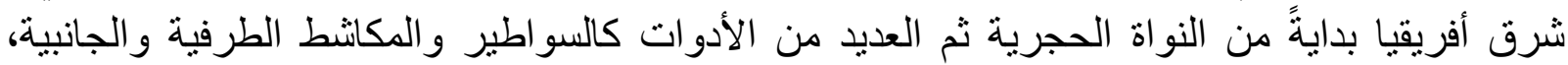

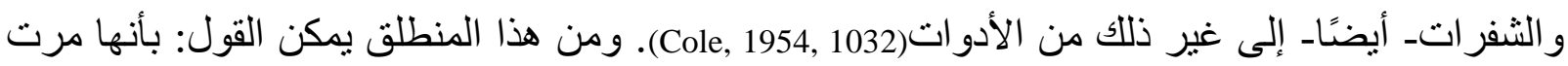
بثلاث مر احل تكنيكية صناعية هي:

Kernel industry .Splinters making صناعة الشظائ . Arrowhead making صناعة النصال

وكان البازلت (حجر زجاجي بركاني أسود) المنحوت موجودًا ابتداءً من العصر الحجري الحديث حتى الحقب التاريخية، ولقد تميز هذا الحجر بإمكانية نحته بسهولة، وكان متو افرًا حول البحر الأحمر في

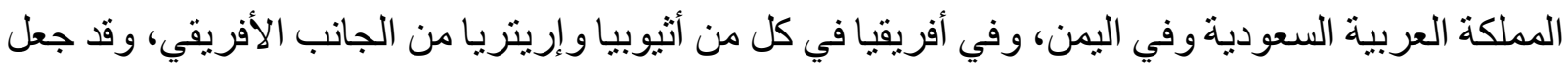

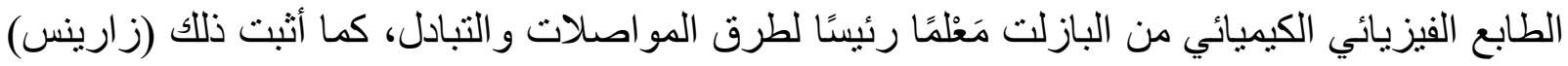

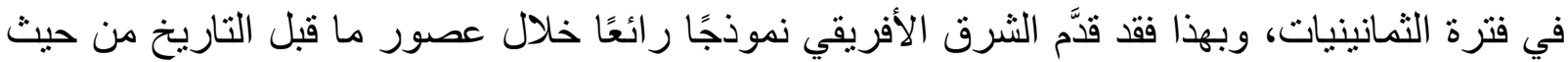

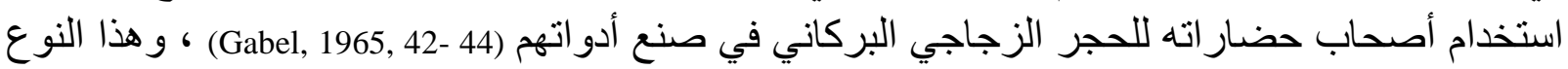
من الأحجار هو ما يعرف داخل شبه الجزيرة العربية باسم الحرات فئه وما زال البازلت المنحوت بالطَرْق وليس بالضغطــ كما درجت العادة في الثرق الأوسط والبحر

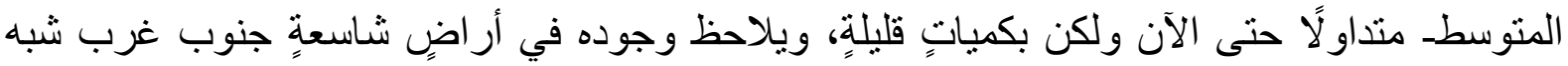
الجزيرة العربية، باعتباره دليلًا على مجموعات حضارية ثقافية قديمة كانت تقطن تللك المناطق (الغبان

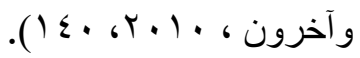




\section{( أ ) موقع ( المندفن ) في جنوب غرب شبه الجزيرة:}

إن منطقة المندفن في شرقي نجر ان، وصحر اء الربع الخالي، قد شهدتا سلسلة متتابعة من البحير ات

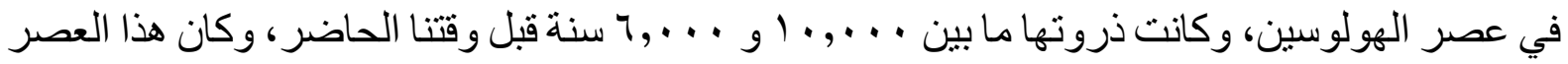

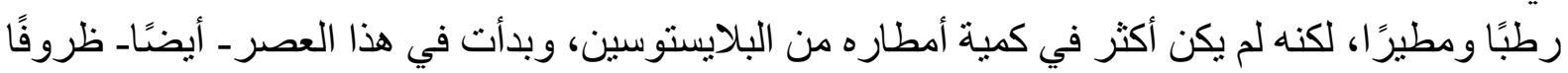
مناخيةً شديدةً من حرارة وجفاف وارتفاع في تكوين الكثبان الرملية، وكانت القطع الأثرية الصوانية الصنية

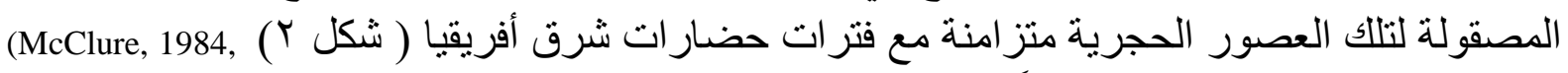

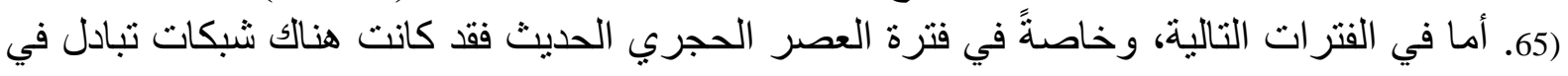
بعض مو اقع جنوب غرب شبه الجزيرة العربية كموقع المندفن هذا (Khalidi, \& Others., 2013, 60). إذن فإن

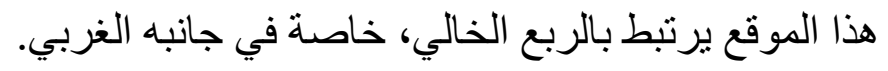

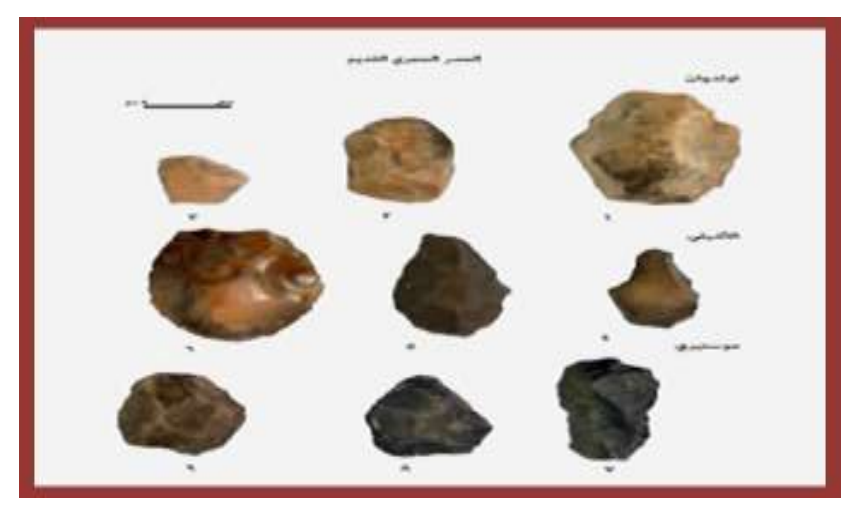

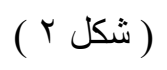

مجمو عة من الشظايا والرقائق و النوى شكلت بأسلوب الليفلو ازي من العصر الحجري القديم الأوسط جمعت من موقع المندفن

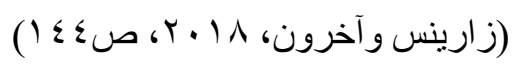

\section{( ) موقع ( الأولدوان ) في شرق أفريقيا:}

أكدت الدر اسات الأثرية أن ثقافات شرق أفريقيا قامت على أكتاف سكان الإقليم الأصليين، ولم بقم

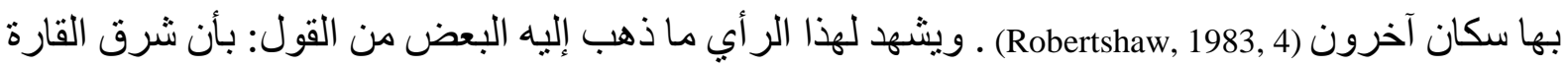

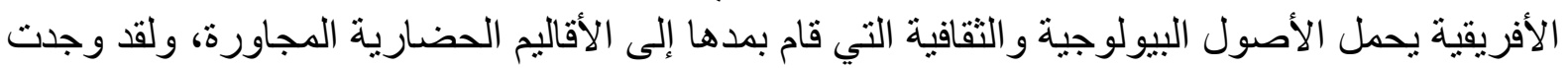

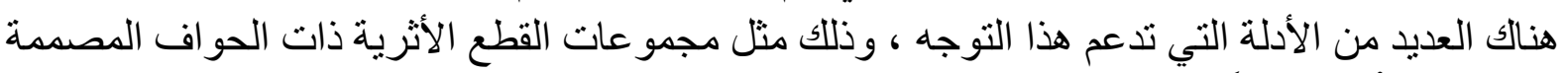

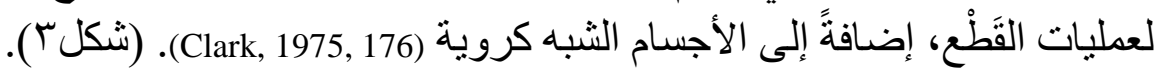


علاقة إقليم جنوب غرب شبه الجزيرة العربية بشرق أفريقيا حتى نهاية العصر الحجري الحديث

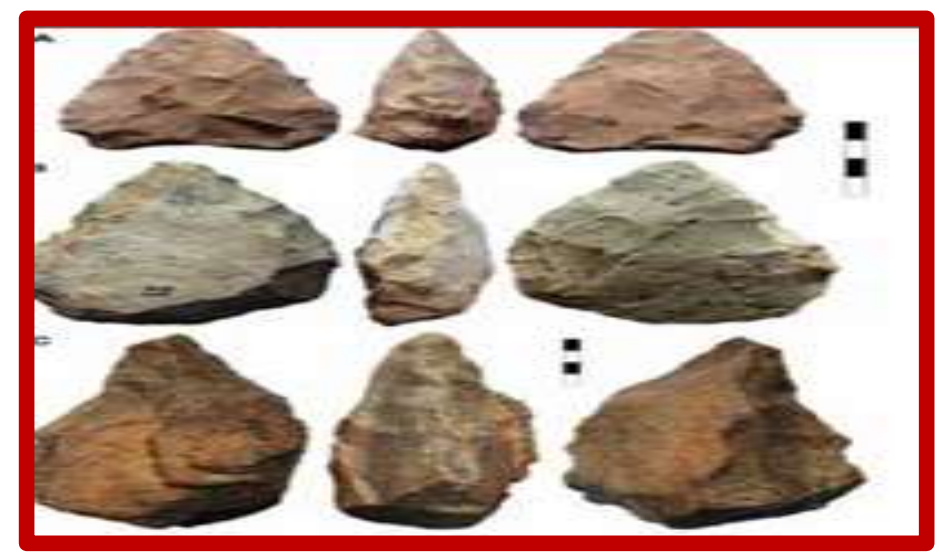

( شكل r)

نماذج لقطع حجرية من الثقافات الأولدو انية والآشولية و الموستيرية التي تعود إلى العصر الحجري القديم في شبه الجزيرة

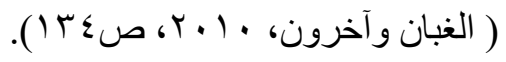

الأولاوان هي أول حضارة عرفها الإنسان من حيث القدم، وقد اكتشفت في مجرى الأولدوفاي في

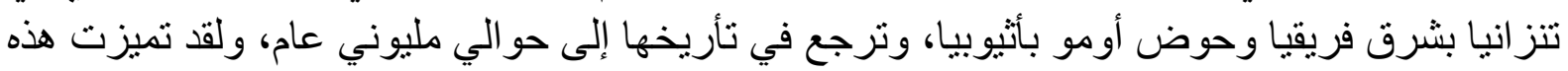

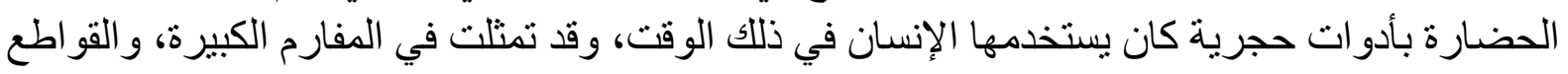

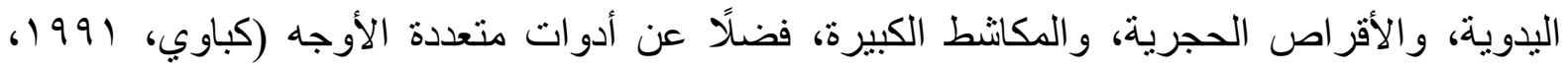

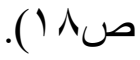

وبالنظر إلى التشابه بين أنماط الأدوات الحجرية الموجودة في جنوب غرب شبه الجزيرة العربية من ناحية التكرار و الحجم مع تلك الموجودة في موقع الأولدوان المتطور في شرق أفريقيا، اعثبر أن هنالك

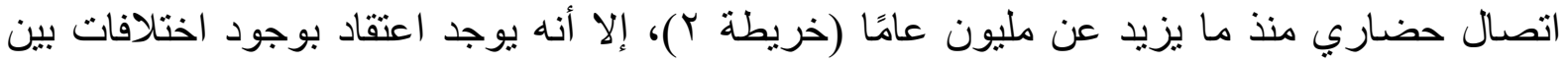

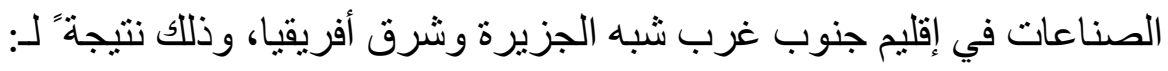

هتخ اخفاء بعض أنماط الصناعات. متر اختلاف طرق الإنتاج، وذلك من حيث استخدام الأساليب التكنولوجية في صناعة الأدوات الحجرية، مثل استخدام النواة الحجرية، في مقابل استخدام عظام الحيو انات الكبيرة في عمليات الطَرق.

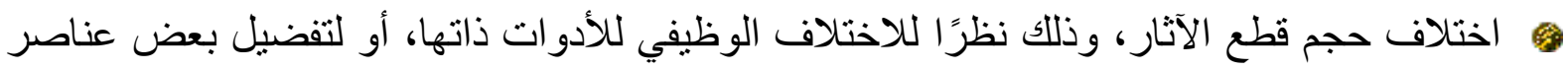

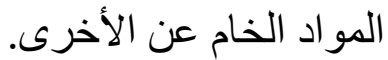

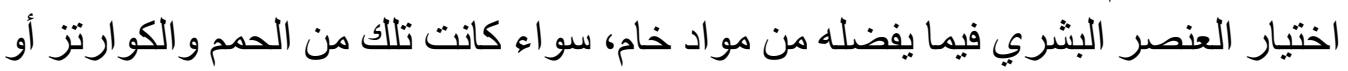

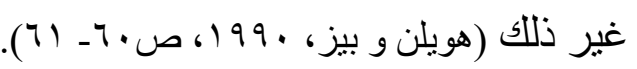

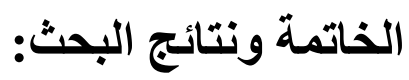

ومما تقدم يرى الباحث أن إقليم جنوب غرب شبه الجزيرة العربية يمثل وحدة مع شرق أفريقيا

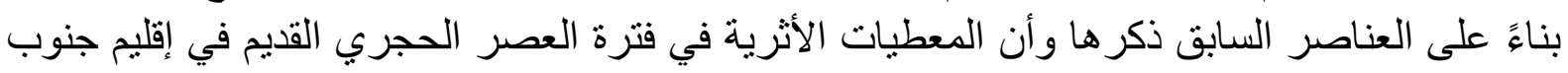

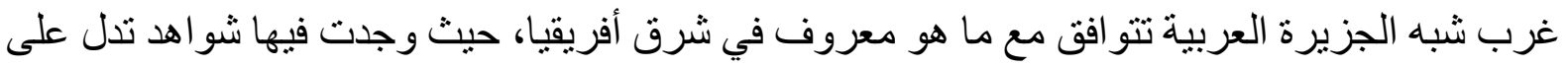


انتشار أقدم السلالات البشرية. وهنا يتضح الدور الذي لعبه إقليم جنوب غرب شبه الجزيرة العربية كمعبر لانتقال الجماعات البشرية المبكرة بين شرقي أفريقيا وآسيا. ويطيب للباحث أن يذكر أهم النتائج التي توصل التهل إلبها من خلال هذه الدراسة.

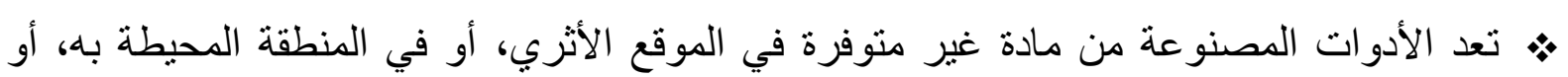

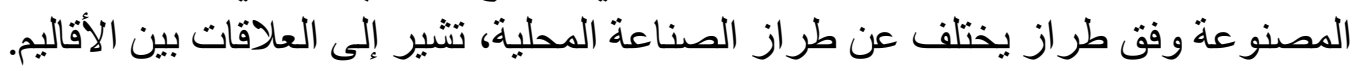

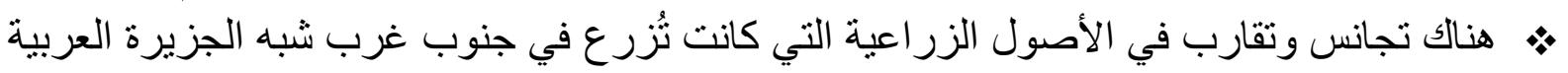

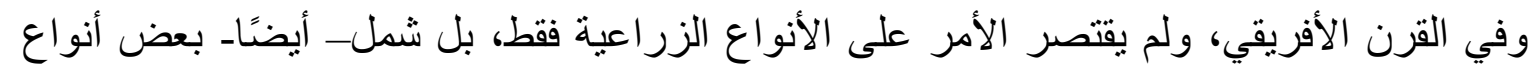

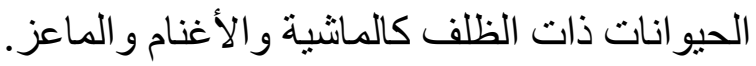
•. رغم الاعتماد على الأدلة الأثرية في تأريخ حياة الإنسان القديم في شرق أفريقيا، إلا أن حياته بكتنفها • استمرت الصناعات الحجرية ذات الطابع النيوليثي في شرق أفريقيا فترة طويلة من الزمن إلى أن حلَّت بعض الغموض. محلها الأدوات المعدنية التي ظهرت في العصور التاحقة.

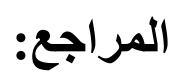

\section{أولًا: المراجع العربية والمعربة:}

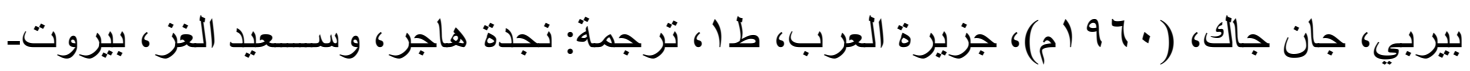

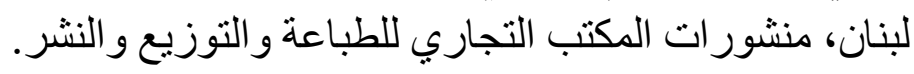

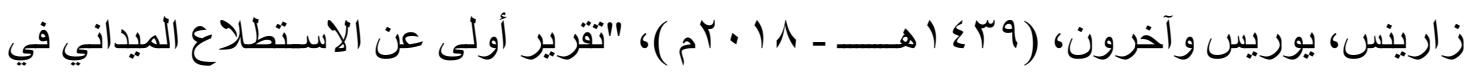

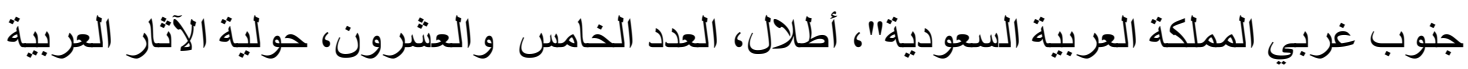

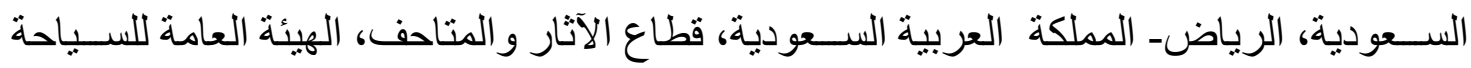

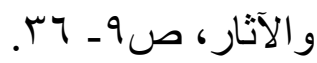

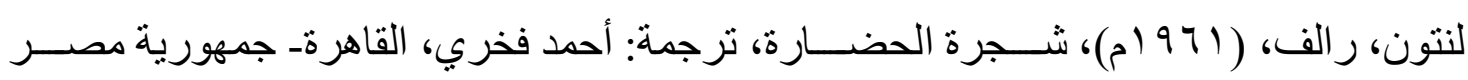
العربية، مطبعة مصر.

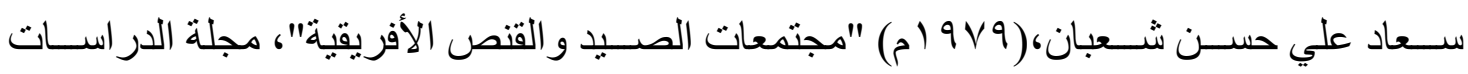

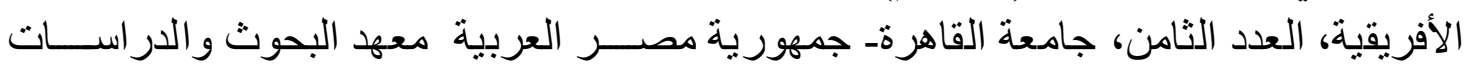

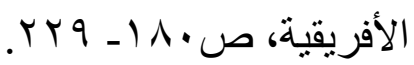

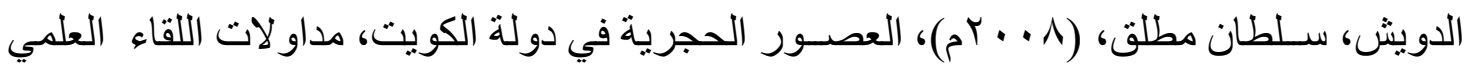

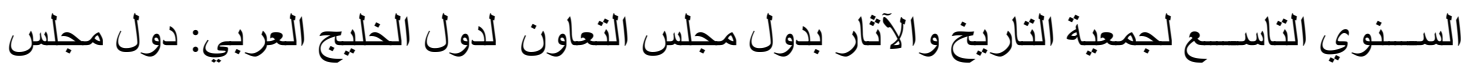
التعاون لدول الخليج العربي عبر العصور ، المملكة العربية السعودية. الناصــري، سـبد أحمد علي، ( •99 (م)، المصــريون و العرب و علاقاتهم بأفريقيا في العصــور القديمة، القاهرة- جمهورية مصر العربية، دار النهضة العبة العربية.

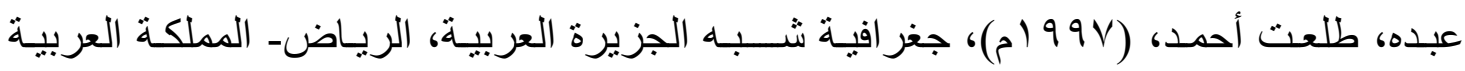
السعودية، دار الخريجي للنشر و التوزيع.

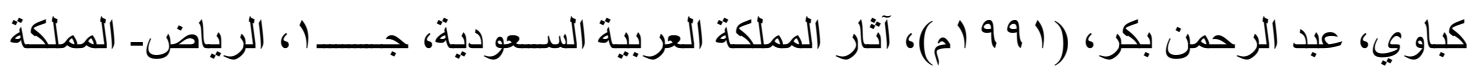

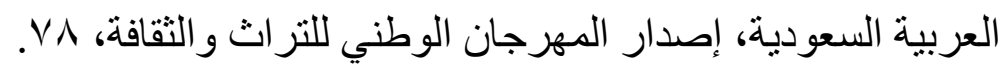

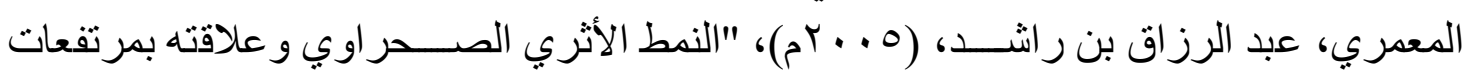


علاقة إقليم جنوب غرب شبه الجزيرة العربية بشرق أفريقيا حتى نهاية العصر الحجري الحديث

جنوبي الجزيرة العربية وبتهامة و عمان"، أدوماتو، العدد الثاني عشـر، الرياضـ المملكة العربية

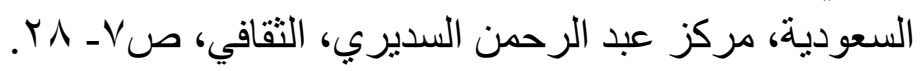

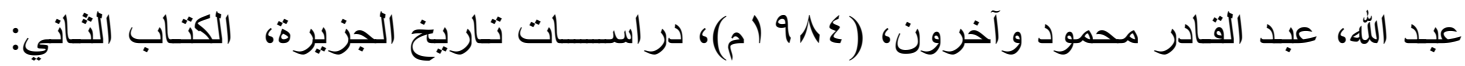

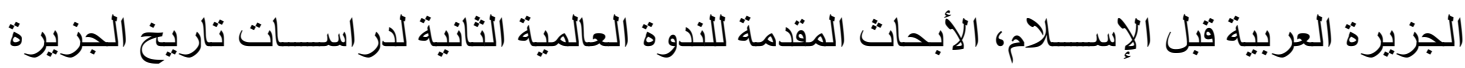

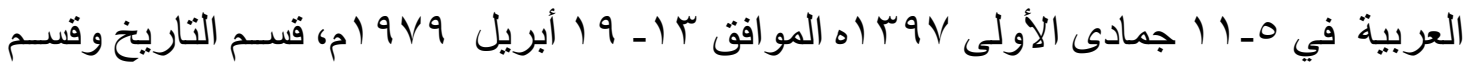
الآثار والمتاحف، كلية الآداب، جامعة الرياض ( جامعة الملك سعود)، الرياض- المملكة العربية السعودية.

الغبان، علي بن إبر اهيم وآخرون، ( • ( • ( م)، طرق التجارة القديمة، روائع آثار المملكة العربية

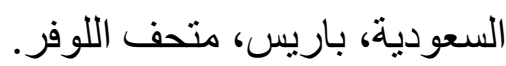

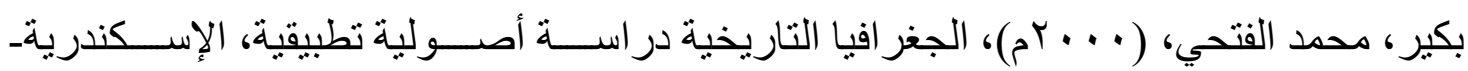
جمهورية مصر العربية، دار المعرفة الجامعية.

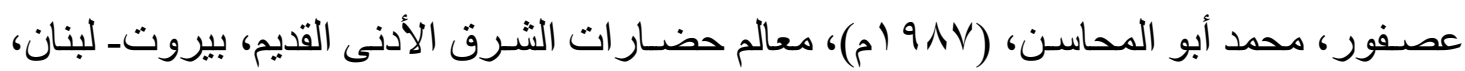
دار النهضة العربية.

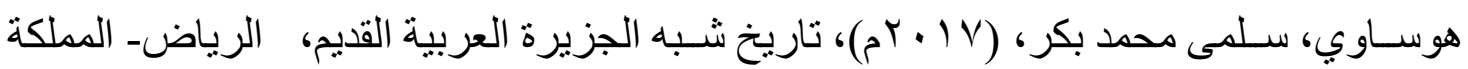

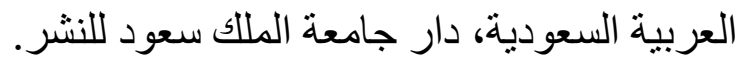

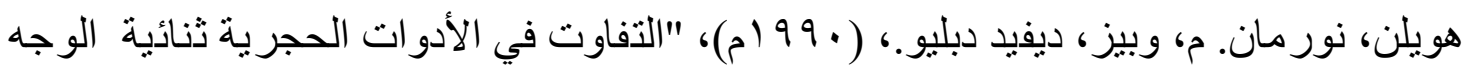

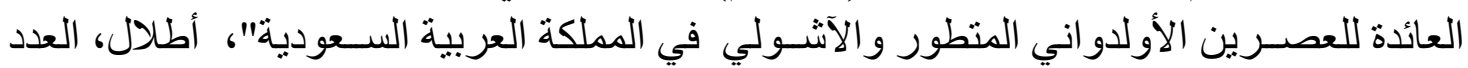

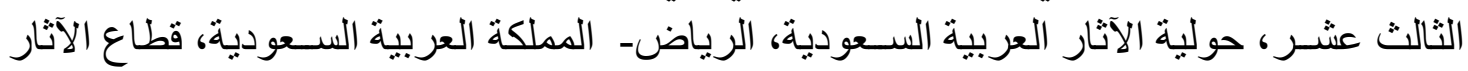

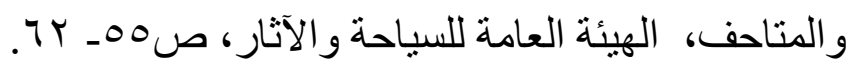

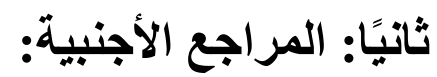

-Andrew, L., (2011), Did Modern Humans Travel .Lawler Out of Africa Via Arabia?.

-Baumann, H., \& Others., (1940), Völkerkunde Von Africa, Essen.

-Beinart, W., (2003), History and African Environments, Oxford.

-Beyin, A., (2006), The Bab al Mandab vs the Nile- Levant: An Appraisal of the Two DispersalRoutes for Early Modern Humans Out of Africa, Afr Archaeol Rev, New York.

-Brown, G. F., (Oct., 1970), Eastern Margin of the Red Sea and the Coastal Structures in Saudi Arabia, PTRS, Vol. 267, No. 1181, A Discussion on the Structure and Evolution of the Red Sea and the Nature of the Red Sea, Gulf of Aden and Ethiopia Rift Junction, pp. 75- 87. 
-Bower, J., (March., 1991)The Pastoral Neolithic of East Africa, JWP, Vol. 5, No. 1, pp. 49- 82.

-Chami, F. A., (2001), Chicken bones from a Neolithic limestone cave site, Zanzibar: Contact between East Africa and Asia, University of Dar-es-Salaam.

-Chami, F. A., \& Amandus, K., (2003), "Neolithic Pottery Traditions from the Islands, the Coast and the Interior of East Africa", AAR, 20, No.2, , pp. 65- 80. -Clark, J. D., (Jun., 1975), Africa in Prehistory: Peripheral or Paramount?, Man, Vol. 10, No. 2, pp. 175- 198.

-Clark, J. D., (1988), He Middle Stone Age of East Africa and the Beginnings of Regional Identity, JWP, Vol. 2, No. 3, pp. 235-305.

-Cole, S., (Dec., 1954) "The Prehistory of East Africa", AA, Vol. 56, No. 6, Part. 1, pp. 1026- 1050.

-David, P., (2005), Human Technical Behavior in the African Middle Stone Age: The Lithic Assemblage of Porc- Epic Cave (Dire Dawa, Ethiopia), AAR, Vol. 22, No. 4, pp. 177- 197.

-Derricourt, R., (2005), Getting "Out of Africa": Sea Crossings, Land Crossings and Culture in the Hominin Migrations, JWP, Vol. 19, No. 2, pp. 119- 132.

-Fuller, D. Q., (2006), "Agricultural Origins and Frontiers in South Asia: A Working Synthesis", JWP, Vol. 20, No. 1, pp. 1- 86.

-Gabel, C., (1965), African Prehistory, BRA, Vol. 4, pp. 40- 83.

-Khalidi, L., \& Others., (2013), Considering the Arabian Neolithic through a reconstitution of interregional obsidian distribution patterns in the region, Arab. Arch. epig, Vol. 24, pp. 59- 67.

-Kusimba, C. M., \& Kusimba, S. B., (June 2005), East African Archaeology, UPMAA, Vol. 22, No. 2, Philadelphia, pp. 105- 109. 
علاقة إقليم جنوب غرب شبه الجزيرة العربية بشرق أفريقيا حتى نهاية العصر الحجري الحديث

-McClure, H., (1984), Late Quaternary Palaeoenvironments of the Rub Al Khali, A thesis submitted for the degree of Doctor of philosophy, in the university of London.

-Michael, D., (2009), The Evolution of Human Populations in Arabia, Research Laboratory for Archaeology University of Oxford.

Mitchell, P., \& Others., (March 2005), Researching Africa's Past: AAR, Vol. 22, No. 1, Oxford University School of Archaeology, pp. 55- 59.

Peninsula: Occupations, Adaptations, and Dispersals, -Petraglia, M. D., , (June., 2003), The Lower Paleolithic of the Arabian JWP, Vol. 17, No. 2, pp. 141- 179.

-Philipson, D., (1993), African Archaeology, Cambridge University Press, Cambridge.

-Robertshaw, P. T., (Jun., 1983), Archaeological Explanation in East Africa, RAIN, No. 56, pp. 4-5.

-Stahl, A. B., (2005), African Archaeology: A Critical Introduction, AAR, Vol. 22, No. 4, pp. 231- 235.

-Tosi, M., (1986), The Emerging Picture of Prehistoric Arabia, ARA, Vil. 15, pp. 461- 490.

-Yosef, O. B., (1987), Pleistocene Connexions between Africa and Southwest Asia: An Archaeological Perspective, AAR, Vol. 5, pp. 29- 38.

-Yosef, O. B., (1994), The Lower Paleolithic of the Near East, JWP, Vol. 8, No. 3, , pp. 211- 265 . 
علاقة إقليم جنوب غرب شبه الجزيرة العربية بشرق أفريقيا حتى نهاية العصر الحجري الحديث

\title{
The relationship of Southwest Arabia with East Africa Until the end of the Neolithic period
}

\author{
Muhammad Taha Muhammad al-Amir
}

PHD Degree - Department of History and Civilization - Division of Ancient History - Faculty of Arabic Language in Cairo - Al-Azhar University Egypt

alamer2951982@gmail.com

Prof. Dr. Aisha Mahmoud Abdalaal

Professor of Egyptology-History

Department

Faculty of Women for Arts, Science \& Education - Ain Shams University - Egypt Aisha_abdelaal@women.asu.edu.eg
Dr. Ahmed Refaat Abd aljawad Assistant Professor of History and Civilization of Egypt and the Ancient Near East - Department of History and Civilization - Faculty of Arabic Language in Cairo - Al-Azhar University - Egypt dr.ahmed.refat2020@gmail.com

\section{Prof. Dr. Talaeat Ahmed Muhammad Abdo}

Professor of Physical Geography - Department of History and Civilization Faculty of Arabic Language in Cairo - Al-Azhar University - Egypt Talaatabdo@gmail.com

\section{Abstract}

The Arabian Peninsula cannot live in a complete isolation in the course of prehistory ages owing to its unique geographical position; it has a close connection with its neighbors inside and outside the Arabian Peninsula.

External communications between the Arabian Peninsula and the neighboring civilizations started during the period between 5000 to 3000 years BC. At a time when the eastern region was strongly connected to the Mesopotamian civilization (Al'ubaid culture), we find that the western region also established early connections with the Nile Valley by land through the Sinai desert, in addition to the sea route, as well as the relations of the southwestern region.

In the Paleolithic age, in its both Oldwaniya and Acholian phases, the archaeological indications belonging to the southwestern region of the Arabian Peninsula conformed with what is known in East Africa where evidence of the spread of the oldest human races was found. Here, we can note the role played by the southwestern Arabian Peninsula as a crossing for the early human population's movement between East Africa and Asia.

Keywords: East Africa - Stone Age - Arabian Peninsula - African Civilization. 